\title{
Off-label-dosing of non-vitamin K-dependent oral antagonists in AF patients before and after stroke: results of the prospective multicenter Berlin Atrial Fibrillation Registry
}

\author{
Serdar Tütüncü ${ }^{1} \cdot$ Manuel Olma $^{1,2}$. Claudia Kunze ${ }^{1}$. Joanna Dietzel ${ }^{1}$. Johannes Schurig ${ }^{1}$. \\ Cornelia Fiessler ${ }^{3}$. Carolin Malsch ${ }^{3,4}$. Tobias Eberhard Haas $^{3}$ - Boris Dimitrijeski ${ }^{5}$. Wolfram Doehner ${ }^{1,6}$. \\ Georg Hagemann ${ }^{7}$. Frank Hamilton ${ }^{8}$. Martin Honermann ${ }^{9}$. Gerhard Jan Jungehulsing ${ }^{10}$. Andreas Kauert $^{11}$. \\ Hans-Christian Koennecke ${ }^{12}$ - Bruno-Marcel Mackert ${ }^{8}$. Darius Nabavi ${ }^{5}$. Christian H. Nolte 1,2,19,20. \\ Joschua Mirko Reis ${ }^{3} \cdot$ Ingo Schmehl $^{13} \cdot$ Paul Sparenberg ${ }^{13} \cdot$ Robert Stingele $^{14} \cdot$ Enrico Völzke $^{15}$. \\ Carolin Waldschmidt ${ }^{16}$. Daniel Zeise-Wehry ${ }^{17}$. Peter U. Heuschmann ${ }^{3,4} \cdot$ Matthias Endress ${ }^{1,2,18,19,20}$. \\ Karl Georg Haeusler ${ }^{21}$
}

Received: 19 July 2021 / Revised: 17 October 2021 / Accepted: 18 October 2021 / Published online: 31 October 2021 (C) The Author(s) 2021

\begin{abstract}
Aims We aimed to analyze prevalence and predictors of NOAC off-label under-dosing in AF patients before and after the index stroke.

Methods The post hoc analysis included 1080 patients of the investigator-initiated, multicenter prospective Berlin Atrial Fibrillation Registry, designed to analyze medical stroke prevention in AF patients after acute ischemic stroke.

Results At stroke onset, an off-label daily dose was prescribed in 61 (25.5\%) of 239 NOAC patients with known AF and $\mathrm{CHA}_{2} \mathrm{DS}_{2}$-VASc score $\geq 1$, of which $52(21.8 \%)$ patients were under-dosed. Under-dosing was associated with age $\geq 80$ years in patients on rivaroxaban [OR 2.90, 95\% CI 1.05-7.9, $P=0.04 ; n=29$ ] or apixaban [OR 3.24, 95\% CI 1.04-10.1, $P=0.04$; $n=22]$. At hospital discharge after the index stroke, NOAC off-label dose on admission was continued in $30(49.2 \%)$ of 61 patients. Overall, 79 (13.7\%) of 708 patients prescribed a NOAC at hospital discharge received an off-label dose, of whom $75(10.6 \%)$ patients were under-dosed. Rivaroxaban under-dosing at discharge was associated with age $\geq 80$ years [OR $3.49,95 \%$ CI 1.24-9.84, $P=0.02 ; n=19$ ]; apixaban under-dosing with body weight $\leq 60 \mathrm{~kg}$ [OR $0.06,95 \%$ CI $0.01-0.47$, $P<0.01 ; n=56$ ], $\mathrm{CHA}_{2} \mathrm{DS}_{2}$-VASc score [OR per point $1.47,95 \% \mathrm{CI} 1.08-2.00, P=0.01$ ], and HAS-BLED score [OR per point $1.91,95 \%$ CI $1.28-2.84, P<0.01]$.

Conclusion At stroke onset, off-label dosing was present in one out of four, and under-dosing in one out of five NOAC patients. Under-dosing of rivaroxaban or apixaban was related to old age. In-hospital treatment after stroke reduced off-label NOAC dosing, but one out of ten NOAC patients was under-dosed at discharge.
\end{abstract}

Clinical trial registration NCT02306824.

Keywords Ischemic stroke $\cdot$ Atrial fibrillation $\cdot$ Under-dosing $\cdot$ NOAC

\section{Introduction}

Randomized controlled trials (RCT) have demonstrated non-inferiority of non-vitamin K-dependent oral anticoagulant (NOAC) compared to the vitamin $\mathrm{K}$ antagonist (VKA)

Karl Georg Haeusler

Haeusler_K@ukw.de

Extended author information available on the last page of the article warfarin in (recurrent) stroke prevention in patients with non-valvular atrial fibrillation (AF) and at least moderate risk of stroke [1-4]. NOACs are superior to warfarin by reducing the risk of intracranial bleeding [5]. Present guidelines strongly recommend oral anticoagulation using a NOAC or a VKA for stroke prevention in patients with $\mathrm{AF}$ and prior ischemic stroke [6-8]. Registry data showed a steep increase of NOAC use for secondary stroke prevention after ischemic stroke/TIA in AF patients, even in elderly stroke patients with AF [5, 9-12]. Treating physicians are 
advised to prescribe the guideline-recommended in-label NOAC dosage in each patient, taking patients' age (for dabigatran, apixaban, and edoxaban), renal function (dabigatran, apixaban, rivaroxaban, and edoxaban) as well as body weight (apixaban and edoxaban) into account [13]. According to prior investigations, up to $50 \%$ of all patients prescribed NOACs were inappropriately dosed, the vast majority of patients being under-dosed [14-17]. This is of clinical relevance, as results from a meta-analysis of randomized controlled trials as well as several registries showed that off-label NOAC dosing is associated with worse clinical outcome [18-20]. Published data on off-label dosing of NOACs is almost exclusively based on AF cohorts including a subset of patients with prior stroke. Findings on AF patients with acute ischemic stroke are rare.

The Berlin Atrial Fibrillation Registry is a multicenter prospective registry including AF patients with acute ischemic stroke/TIA. First results on factors associated with oral anticoagulation use were published previously [9]. In the present analysis, we focus on prevalence and predictors of NOAC under-dosing in $\mathrm{AF}$ patients before and after the index stroke.

\section{Methods}

\section{Design of the registry}

The design of the "Berlin Atrial Fibrillation Registry" was described in detail previously [9]. Patients with acute ischemic stroke/TIA, admitted to 1 of the 16 stroke units in Berlin, Germany, were eligible for inclusion, if patients were able to give informed consent within the in-hospital stay and either had a history of AF or a first episode of AF in-hospital. Conformation of stroke/TIA was based on brain imaging and clinical criteria, following the WHO definition [21]. No treatment recommendations were given to treating physicians at local sites.

\section{Data assessment}

Collected information included patients' demographics, past medical history, AF type, stroke type, individual stroke treatment, stroke severity (assessed by the National Institutes of Health Stroke Scale (NIHSS) score[22] on admission and the modified Rankin Scale (mRS) score at discharge[23]), body weight, renal function and antithrombotic medication on admission as well as at hospital discharge. The $\mathrm{CHA}_{2} \mathrm{DS}_{2}$-VASc and HAS-BLED score were calculated according to the current ESC guideline [6]. NOAC off-label dosing was defined according to guideline recommendations and the EHRA practical guide $[6,7,13]$. As no serumcreatinine or eGFR value was documented by the treating physicians, two patients received rivaroxaban before stroke onset and three patients after stroke were excluded from this analysis. Furthermore, two patients on apixaban at discharge were excluded because of missing values relevant to categorize regarding in- or off-label dosing (serum-creatinine level or body weight).

\section{Statistical methods}

Statistical analysis was performed in cooperation with the Institute for Clinical Epidemiology and Biometry at the Julius-Maximilians-University of Würzburg, Germany. Baseline characteristics of study patients are presented as absolute and relative frequencies or median and quartiles. A two-sided Pearson $\chi^{2}$ test or Mann-Whitney $U$ test was performed for univariate analyses. Analysis of predictors for the main outcomes rivaroxaban or apixaban under-dosed at stroke onset and at discharge was conducted using multiple binary logistic regression analysis and adjusted odds ratios (OR) and respective confidence intervals (CI) are reported. Parameters with $P$ values $<0.05$ in univariate analysis were transferred into multiple binary logistic regression analysis and variable selection was conducted using backward selection algorithm. For comparability of results, we adjusted the analysis of predictors for rivaroxaban for predictors of apixaban, and vice versa. The same strategy was applied for the multiple analyses regarding under-dosed rivaroxaban or apixaban at discharge. As sensitivity analysis, we pooled the datasets of rivaroxaban and apixaban and calculated multiple logistic regression models with outcome "rivaroxaban or apixaban" at stroke onset and at hospital discharge. In these models, variables with $P$ values $<0.05$ in multivariate analyses of Tables 1 and 2 or Tables 3 and 4 were included, respectively. Statistical analysis was performed using SPSS (Version 24, SPSS Inc., Chicago, USA).

\section{Results}

Derivation of study population is shown in Fig. 1 online supplement. Baseline characteristics of the analyzed cohorts are presented in Table 1 online supplement. Of 1080 registry patients, 36 were excluded due to violation of in- or exclusion criteria, $753(69.7 \%)$ of 1044 patients had known AF and a $\mathrm{CHA}_{2} \mathrm{DS}_{2}$-VASc score $\geq 1$ pre-stroke. Of these 753 patients, $466(61.9 \%)$ had been anticoagulated at stroke onset [227 (30.1\%) VKA, 38 (5.0\%) dabigatran, 131 (17.4\%) rivaroxaban, $64(8.5 \%)$ apixaban, and $6(0.8 \%)$ edoxaban].

\section{NOAC dosing at stroke onset in patients with known AF}

An off-label daily dose was prescribed in 35 (26.7\%) of 131 patients on rivaroxaban, with $6(4.5 \%)$ patients being 
Table 1 Characteristics of 124 registry patients with known AF and rivaroxaban prescription at stroke onset with regard to on-label dosing or off-label under-dosing

\begin{tabular}{|c|c|c|c|c|c|}
\hline & \multicolumn{3}{|c|}{ Univariate analysis } & \multicolumn{2}{|c|}{ Multivariate analysis } \\
\hline & On-label dosing & Under-dosing & $P$ value & OR $[95 \% \mathrm{CI}]^{*}$ & $P$ value \\
\hline$n$ & 95 & 29 & & & \\
\hline Age, median [IQR] & 77 [71-83] & 81 [76-87] & 0.02 & - & - \\
\hline Age $\geq 80$ years, $n(\%)$ & $35(36.8)$ & $18(62.1)$ & 0.02 & $2.90[1.05-8.00]$ & 0.04 \\
\hline Sex, male, $n(\%)$ & $50(52.6)$ & $12(41.4)$ & 0.30 & - & - \\
\hline Body weight, median [IQR] & 80 [70-90] & $72[62-82]$ & 0.02 & $0.97[0.94-1.00]$ & 0.07 \\
\hline Body weight $\leq 60 \mathrm{~kg}, n(\%)$ & $11(11.6)$ & $5(17.9)$ & 0.39 & - & - \\
\hline Index event TIA, $n(\%)$ & $36(37.9)$ & $7(24.1)$ & 0.173 & - & - \\
\hline Prior stroke/TIA, $n(\%)$ & $35(36.8)$ & $9(31.0)$ & 0.60 & - & - \\
\hline Hypertension, $n(\%)$ & $83(87.4)$ & $27(93.1)$ & 0.40 & - & - \\
\hline Congestive heart failure, $n(\%)$ & $15(15.8)$ & $4(13.0)$ & 0.80 & - & - \\
\hline Diabetes, $n(\%)$ & $32(33.7)$ & $11(37.9)$ & 0.67 & - & - \\
\hline Vascular disease, $n(\%)$ & $26(27.4)$ & $13(44.8)$ & 0.08 & - & - \\
\hline eGFR on admission, $\mathrm{ml} / \mathrm{min} / 1.72 \mathrm{~m}^{2}$, median $[\mathrm{IQR}]$ & $65[50-80]$ & $63[55-71]$ & 0.97 & $1.01[0.98-1.05]$ & 0.41 \\
\hline $\mathrm{eGFR}=15-49 \mathrm{ml} / \mathrm{min} / 1.72 \mathrm{~m}^{2}, n(\%)$ & $25(26.3)$ & $0(0.0)$ & $<0.01$ & - & - \\
\hline $\mathrm{CHA}_{2} \mathrm{DS}_{2}$-VASc pre-stroke, median [IQR] & $5.0[3.0-6.0]$ & $5.0[4.0-6.0]$ & 0.27 & $1.35[0.90-2.02]$ & 0.15 \\
\hline HAS-BLED pre-stroke, median [IQR] & $2.0[2.0-3.0]$ & $2.0[2.0-3.0]$ & 0.43 & $0.56[0.31-1.01]$ & 0.05 \\
\hline
\end{tabular}

* OR and $95 \% \mathrm{CI}$ for continuous variables were expressed per point (body weight, $\mathrm{CHA}_{2} \mathrm{DS}_{2}$-VASc pre-stroke, HAS-BLED pre-stroke) or per unit $\mathrm{ml} / \mathrm{min} / 1.72 \mathrm{~m}^{2}$ (eGFR on admission)

Table 2 Characteristics of 61 registry patients with known AF and apixaban prescription at stroke onset with regard to on-label or off-label under-dosing

\begin{tabular}{|c|c|c|c|c|c|}
\hline & \multicolumn{3}{|c|}{ Univariate analysis } & \multicolumn{2}{|c|}{ Multivariate analysis } \\
\hline & On-label dosing & Under-dosing & $P$ value & OR $[95 \% \mathrm{CI}]^{*}$ & $P$ value \\
\hline$n$ & 39 & 22 & & & \\
\hline Age, median [IQR] & 77 [71-85] & $82[75-86]$ & 0.16 & - & - \\
\hline Age $\geq 80$ years, $n(\%)$ & $17(43.6)$ & $15(68.2)$ & 0.07 & $3.24[1.04-10.1]$ & 0.04 \\
\hline Sex, male, $n(\%)$ & $25(64.1)$ & $11(50.0)$ & 0.28 & - & - \\
\hline Body weight, median [IQR] & 75 [66-87] & 77 [66-83] & 0.96 & $1.02[0.98-1.07]$ & 0.35 \\
\hline Body weight $\leq 60 \mathrm{~kg}, n,(\%)$ & $6(15.4)$ & $0(0.0)$ & 0.06 & - & - \\
\hline Index event TIA, $n(\%)$ & $18(46.2)$ & $9(40.9)$ & 0.69 & - & - \\
\hline Prior stroke/TIA, $n(\%)$ & $22(56.4)$ & $10(45.5)$ & 0.41 & - & - \\
\hline Hypertension, $n(\%)$ & $35(89.7)$ & $21(95.5)$ & 0.44 & - & - \\
\hline Congestive heart failure, $n(\%)$ & $6(15.4)$ & $4(18.2)$ & 0.78 & - & - \\
\hline Diabetes, $n(\%)$ & $12(30.8)$ & $10(45.5)$ & 0.25 & - & - \\
\hline Vascular disease, $n(\%)$ & $15(38.5)$ & $12(54.5)$ & 0.23 & - & - \\
\hline eGFR on admission, $\mathrm{ml} / \mathrm{min} / 1.72 \mathrm{~m}^{2}$, median [IQR] & $66[49-81]$ & $52[45-62]$ & 0.10 & $0.98[0.95-1.01]$ & 0.20 \\
\hline eGFR $15-29 \mathrm{ml} / \mathrm{min} / 1.72 \mathrm{~m}^{2}, n(\%)$ & $4(10.3)$ & $0(0.0)$ & 0.12 & - & - \\
\hline Serum creatinine, $\geq 1.5 \mathrm{mg} / \mathrm{dl}, n(\%)$ & $8(20.5)$ & $2(9.1)$ & 0.25 & - & - \\
\hline $\mathrm{CHA}_{2} \mathrm{DS}_{2}$-VASc pre-stroke, median [IQR] & $5[4-6]$ & $5[4-6]$ & 0.35 & $0.97[0.57-1.63]$ & 0.90 \\
\hline HAS-BLED pre-stroke, median [IQR] & $3[2,3]$, & $3[2-4]$ & 0.29 & $1.27[0.66-2.44]$ & 0.48 \\
\hline
\end{tabular}

*OR and $95 \% \mathrm{CI}$ for continuous variables were expressed per point (body weight, $\mathrm{CHA}_{2} \mathrm{DS}_{2}$-VASc pre-stroke, HAS-BLED pre-stroke) or per unit $\mathrm{ml} / \mathrm{min} / 1.72 \mathrm{~m}^{2}$ (eGFR on admission) 
Table 3 Characteristics of 104 registry patients with rivaroxaban at hospital discharge after the index stroke with regard to prescription of onlabel or off-label under-dosing

\begin{tabular}{|c|c|c|c|c|c|}
\hline & \multicolumn{3}{|c|}{ Univariate analysis } & \multicolumn{2}{|c|}{ Multivariate analysis } \\
\hline & On-label dosing & Under-dosing & $P$ value & OR $[95 \% \mathrm{CI}]^{*}$ & $P$ value \\
\hline$n$ & 85 & 19 & & & \\
\hline Age, median [IQR] & $76[71-81]$ & $84[74-88]$ & $<0.01$ & - & - \\
\hline Age $\geq 80$ years, $n(\%)$ & $28(32.9)$ & $12(63.2)$ & 0.01 & $3.50[1.24-9.84]$ & 0.02 \\
\hline Sex, male, $n(\%)$ & $42(49.4)$ & $9(47.4)$ & 0.87 & - & - \\
\hline Body weight, median [IQR] & 80 [70-90] & $70[64-80]$ & 0.04 & $0.98[0.93-1.02]$ & 0.25 \\
\hline Body weight $<60 \mathrm{~kg}, n(\%)$ & $10(11.8)$ & $3(15.8)$ & 0.63 & - & - \\
\hline Index event TIA, $n(\%)$ & $34(40.0)$ & $6(31.6)$ & 0.50 & - & - \\
\hline NIHSS on admission, median [IQR] & $2.0[1.0-4.0]$ & $2.0[1.0-3.0]$ & 0.82 & - & - \\
\hline NIHSS on admission, categories, $n(\%)$ & & & 0.34 & - & - \\
\hline $0-3$ & $57(67.1)$ & $15(78.9)$ & - & - & - \\
\hline $4-7$ & $20(23.5)$ & $4(21.1)$ & - & - & - \\
\hline$\geq 8$ & $8(9.4)$ & $0(0.0)$ & - & - & - \\
\hline mRS at discharge, median [IQR] & $2.0[1.0-3.0]$ & $2.0[1.0-3.0]$ & 0.59 & - & - \\
\hline $\mathrm{mRS}$ at discharge, categories, $n(\%)$ & & & 0.34 & - & - \\
\hline $0-1$ & $35(41.2)$ & $5(26.3)$ & - & - & - \\
\hline $2-3$ & $30(35.3)$ & $10(52.6)$ & - & - & - \\
\hline $4-5$ & $20(23.5)$ & $4(21.1)$ & - & - & - \\
\hline Intravenous thrombolysis, $n(\%)$ & $1(1.2)$ & $1(5.3)$ & 0.24 & - & - \\
\hline Carotid endarterectomy, $n(\%)$ & $0(0.0)$ & $0(0.0)$ & 1.00 & - & - \\
\hline Endovascular thrombectomy, $n(\%)$ & $2(2.4)$ & $0(0.0)$ & 1.00 & - & - \\
\hline First episode of AF in-hospital, $n(\%)$ & $9(10.6)$ & $0(0.0)$ & 0.14 & - & - \\
\hline Prior stroke/TIA, $n(\%)$ & $32(37.6)$ & $5(26.3)$ & 0.35 & - & - \\
\hline Hypertension, $n(\%)$ & $72(84.7)$ & $17(89.5)$ & 0.59 & - & - \\
\hline Congestive heart failure, $n(\%)$ & $15(17.6)$ & $5(26.3)$ & 0.39 & $1.56[0.45-5.33]$ & 0.49 \\
\hline Diabetes, $n(\%)$ & $32(37.6)$ & $5(26.3)$ & 0.35 & - & - \\
\hline Vascular disease, $n(\%)$ & $29(34.1)$ & $5(26.3)$ & 0.51 & - & - \\
\hline eGFR at discharge, $\mathrm{ml} / \mathrm{min} / 1.72 \mathrm{~m}^{2}$ median [IQR] & $66[53-78]$ & 65 [57-74] & 0.71 & $1.02[0.98-1.05]$ & 0.34 \\
\hline eGFR $15-49 \mathrm{ml} / \mathrm{min} / 1.72 \mathrm{~m}^{2}, n(\%)$ & $16(18.8)$ & $0(0.0)$ & 0.04 & - & - \\
\hline $\mathrm{CHA}_{2} \mathrm{DS}_{2}$-VASc post-stroke, median [IQR] & $6.0[5.0-7.0]$ & $6.0[5.0-6.0]$ & 0.84 & $0.89[0.52-1.51]$ & 0.66 \\
\hline HAS-BLED post-stroke, median [IQR] & $3.0[3.0-3.0]$ & $3.0[3.0-3.0]$ & 0.56 & $1.23[0.53-2.87]$ & 0.63 \\
\hline
\end{tabular}

*OR and $95 \% \mathrm{CI}$ for continuous variables were expressed per point (body weight, $\mathrm{CHA}_{2} \mathrm{DS}_{2}$-VASc post-stroke, HAS-BLED post-stroke) or per unit $\mathrm{ml} / \mathrm{min} / 1.72 \mathrm{~m}^{2}(\mathrm{eGFR}$ at discharge)

over-dosed (receiving $20 \mathrm{mg}$ OD instead of $15 \mathrm{mg}$ OD), and $29(22.1 \%)$ patients being under-dosed (receiving $10 \mathrm{mg}$ OD $(n=1)$ or $15 \mathrm{mg}$ OD $(n=28)$ instead of $20 \mathrm{mg}$ OD). Rivaroxaban under-dosing was associated with age $\geq 80$ years [OR $2.90,95 \%$ CI 1.05-7.9, $P=0.04$ ] (Table 1). An off-label daily dose of apixaban was prescribed in $25(39.1 \%)$ of 64 patients, with 3 (4.7\%) patients being over-dosed (receiving $5.0 \mathrm{mg}$ TD instead of $2.5 \mathrm{mg} \mathrm{TD}$ ), and 22 (34.4\%) patients being underdosed (receiving $2.5 \mathrm{mg}$ TD instead of $5 \mathrm{mg}$ TD). Apixaban under-dosing was associated with age $\geq 80$ years [OR $3.24,95 \%$ CI 1.04-10.1, $P=0.04$ ] (Table 2). Taken together, $60(30.8 \%)$ of 195 patients on apixaban or rivaroxaban had an off-label daily dose at stroke onset, with 51 (26.2\%) patients being under-dosed. Under-dosing of rivaroxaban or apixaban was associated with age $\geq 80$ years [OR 2.97, 95\% CI 1.50-5.88, $P<0.01$ ] (Table 2 online supplement).

Edoxaban was dosed according to label in all six patients at stroke onset. One $(2.6 \%)$ out of 38 patients on dabigatran was under-dosed (receiving $75 \mathrm{mg}$ TD). According to recent recommendations, an inappropriate daily dose of dabigatran was prescribed in $9(23.7 \%)$ of 38 patients (receiving $110 \mathrm{mg}$ TD instead of $150 \mathrm{mg}$ TD). Prescription of dabigatran $110 \mathrm{mg}$ TD vs. $150 \mathrm{mg}$ TD at the time of stroke was associated with age $\geq 80$ years $[0 \%$ vs. $34.6 \%, P=0.04$ ] (Table 5 online supplement). In summary, 61 (25.5\%) of 239 patients prescribed a NOAC had an off-label daily dose at stroke onset, with 52 (21.8\%) patients being under-dosed. 
Table 4 Characteristics of 438 registry patients with apixaban at hospital discharge after the index stroke with regard to prescription of on-label or off-label under-dosing

\begin{tabular}{|c|c|c|c|c|c|}
\hline & Univariate analys & & & Multivariate analy & \\
\hline & On-label dosing & Under-dosing & $P$ value & OR $[95 \% \mathrm{CI}]$ & $P$ value \\
\hline$n$ & 382 & 56 & & & \\
\hline Age, median [IQR] & 77 [72-82] & $81[78-86]$ & $<0.01$ & - & - \\
\hline Age $\geq 80$ years, $n(\%)$ & $147(38.5)$ & $35(62.5)$ & $<0.01$ & $1.85[0.98-3.49]$ & 0.06 \\
\hline Sex, male, $n(\%)$ & $203(53.1)$ & $28(50.0)$ & 0.66 & & \\
\hline Body weight, median [IQR] & $78[67-88]$ & $74[68-80]$ & 0.41 & & \\
\hline Body weight $\leq 60 \mathrm{~kg}, n(\%)$ & $56(14.7)$ & $1(1.8)$ & $<0.01$ & $0.06[0.01-0.47]$ & $<0.01$ \\
\hline Index event TIA, $n(\%)$ & $83(21.7)$ & $13(23.2)$ & 0.80 & - & - \\
\hline NIHSS on admission (median [IQR]) & $2.0[1.0-5.0]$ & $3.0[1.0-6.0]$ & 0.44 & - & - \\
\hline NIHSS on admission, $(n)(\%)$ & & & 0.24 & - & - \\
\hline $0-3$ & $242(63.4)$ & $29(51.8)$ & - & - & - \\
\hline $4-7$ & $92(24.1)$ & $17(30.4)$ & - & - & - \\
\hline$\geq 8$ & $48(12.6)$ & $10(17.9)$ & - & - & - \\
\hline mRS at discharge (median [IQR]) & $2.0[1.0-3.0]$ & $3.0[1.0-4.0]$ & 0.20 & - & - \\
\hline $\mathrm{mRS}$ at discharge, categories, $n(\%)$ & & & 0.21 & - & - \\
\hline $0-1$ & $113(29.6)$ & $15(26.8)$ & - & - & - \\
\hline $2-3$ & $186(48.7)$ & $23(41.1)$ & - & - & - \\
\hline $4-5$ & $83(21.7)$ & $18(32.1)$ & - & - & - \\
\hline Intravenous thrombolysis, $n(\%)$ & $69(18.1)$ & $11(19.6)$ & 0.78 & - & - \\
\hline Carotid endarterectomy, $n(\%)$ & $2(0.5)$ & $1(1.8)$ & 0.29 & - & - \\
\hline Endovascular thrombectomy, $n(\%)$ & $33(8.6)$ & $4(7.1)$ & 0.71 & - & - \\
\hline First episode of AF in-hospital, $n(\%)$ & $147(38.5)$ & $13(23.1)$ & 0.03 & $0.63[0.31-1.27]$ & 0.19 \\
\hline Prior stroke/TIA, $n(\%)$ & $108(28.3)$ & $18(32.1)$ & 0.55 & - & - \\
\hline Hypertension, $n(\%)$ & $339(88.7)$ & $52(92.9)$ & 0.35 & - & - \\
\hline Congestive heart failure, $n(\%)$ & $39(10.2)$ & $12(21.4)$ & 0.02 & $1.17[0.47-2.90]$ & 0.74 \\
\hline Diabetes, $n(\%)$ & $99(25.9)$ & $17(30.4)$ & 0.48 & - & - \\
\hline Vascular disease, $n(\%)$ & $98(25.7)$ & $20(35.7)$ & 0.11 & - & - \\
\hline eGFR at discharge, $\mathrm{ml} / \mathrm{min} / 1.72 \mathrm{~m}^{2}$ median [IQR] & $66[54-81]$ & 56 [44-69] & $<0.01$ & $0.99[0.97-1.01]$ & 0.16 \\
\hline eGFR $15-29 \mathrm{ml} / \mathrm{min} / 1.72 \mathrm{~m}^{2}, n(\%)$ & $10(2.6)$ & $0(0.0)$ & 0.22 & - & - \\
\hline Serum creatinine $\geq 1.5 \mathrm{mg} / \mathrm{dl}, n(\%)$ & $32(8.4)$ & $6(10.7)$ & 0.56 & - & - \\
\hline $\mathrm{CHA}_{2} \mathrm{DS}_{2}$-VASc post-stroke, median [IQR] & $6.0[5.0-6.0]$ & $6.0[6.0-7.0]$ & $<0.01$ & $1.47[1.08-2.00]$ & 0.01 \\
\hline HAS-BLED post-stroke, median [IQR] & $3.0[3.0-4.0]$ & $4.0[3.0-4.0]$ & $<0.01$ & $1.91[1.28-2.84]$ & $<0.01$ \\
\hline
\end{tabular}

*OR and $95 \% \mathrm{CI}$ for continuous variables were expressed per point $\left(\mathrm{CHA}_{2} \mathrm{DS}_{2}\right.$-VASc post-stroke, HAS-BLED post-stroke) or per unit ml/ $\mathrm{min} / 1.72 \mathrm{~m}^{2}$ (eGFR at discharge

There was not a significant impact of rivaroxaban or apixaban under-dosing vs. on-label dosing on severity of the index stroke/TIA (assessed by the NIHSS or mRS) as depicted in Table 7 Online Supplement.

\section{Adaption of treatment in patients with known AF and off-label NOAC dose at stroke onset}

Out of six patients on rivaroxaban over-dosed at stroke onset, treating physicians switched antithrombotic medication in two patients and maintained the $20 \mathrm{mg}$ OD dose in four patients (eGFR improved to $\geq 50 \mathrm{ml} / \mathrm{min}$ in one patient in-hospital). In 29 patients on rivaroxaban who were under-dosed at stroke onset, treating physicians switched antithrombotic medication in 10 patients, adapted the dose in 6 patients and maintained the $15 \mathrm{mg}$ OD dose in 13 patients (eGFR declined to $<50 \mathrm{ml} / \mathrm{min}$ in 1 patient in-hospital). Subsequently, off-label rivaroxaban dosing at stroke onset was continued at hospital discharge in 15 (42.9\%) out of 35 patients. In all three patients on apixaban over-dosed at stroke onset, the $5 \mathrm{mg}$ TD dose was maintained. In 22 patients on apixaban under-dosed at stroke onset, treating physicians adapted the dose according to label in 10 patients and maintained the $2.5 \mathrm{mg}$ TD dose in 12 patients (with creatinine remained $<1.5 \mathrm{mg} / \mathrm{dl}$ inhospital). Subsequently, off-label apixaban dosing at stroke 
onset was continued at hospital discharge in $15(60.0 \%)$ out of 25 patients. One patient receiving dabigatran $75 \mathrm{mg}$ TD at stroke onset was discharged on (under-dosed) apixaban.

\section{NOAC dosing at hospital discharge after acute ischemic stroke or TIA}

At hospital discharge, 843 (81.2\%) of 1,038 registry patients with either known AF or a first episode of AF in-hospital were anticoagulated [135 (16.0\%) on VKA, 134 (15.9\%) on dabigatran, $111(13.2 \%)$ on rivaroxaban, $459(54.5 \%)$ on apixaban, and $4(0.5 \%)$ on edoxaban], including $616(81.3 \%)$ of 758 registry patients with known AF [126 (16.6\%) on VKA, $96(12.7 \%)$ on dabigatran, $100(13.2 \%)$ on rivaroxaban, $292(38.5 \%)$ on apixaban, and $2(0.3 \%)$ on edoxaban], and 227 (79.4\%) of 286 registry patients with a first episode of AF in-hospital [9 (3.1\%) on VKA, $38(13.3 \%)$ on dabigatran, $11(3.8 \%)$ on rivaroxaban, $167(58.4 \%)$ on apixaban, and $2(0.7 \%)$ on edoxaban]. An off-label dose was prescribed in $97(13.7 \%)$ of 708 NOAC patients, including 79 (11.2\%) with known AF and 18 (2.5\%) with a first episode of AF inhospital $(P<0.01)$. In other terms, an off-label dose was prescribed in $79(16.1 \%)$ of 490 with known AF and 18 (8.2\%) of 218 NOAC patients with a first episode of AF in-hospital.

An off-label daily dose was prescribed in 23 (20.7\%) of $111 \mathrm{AF}$ patients on rivaroxaban, with $5(4.5 \%)$ patients being over-dosed [receiving $20 \mathrm{mg}$ OD instead of $15 \mathrm{mg}$ OD $(n=4)$ or $30 \mathrm{mg}$ OD instead of $20 \mathrm{mg}$, and $19(17.1 \%)$ patients being under-dosed (receiving $15 \mathrm{mg}$ OD instead of $20 \mathrm{mg}$ OD). Rivaroxaban under-dosing at hospital discharge after stroke was associated with age $\geq 80$ years [OR 3.49, 95\% CI 1.24-9.84, $P=0.02$ ] (Table 3).

An off-label daily dose was prescribed in $74(16.1 \%)$ patients of $459 \mathrm{AF}$ patients on apixaban, with 18 (3.9\%) patients being over-dosed (receiving $5.0 \mathrm{mg}$ TD instead of $2.5 \mathrm{mg} \mathrm{TD}$ ), and with 56 (12.2\%) patients being underdosed (receiving $2.5 \mathrm{mg}$ TD instead of $5 \mathrm{mg}$ TD). Apixaban under-dosing at hospital discharge after stroke was associated with body weight $\leq 60 \mathrm{~kg}$ [OR $0.06,95 \%$ CI $0.01-0.47$, $P<0.01$ ], $\mathrm{CHA}_{2} \mathrm{DS}_{2}$-VASc score [OR per point $1.47,95 \%$ CI 1.08-2.00, $P=0.01$ ], and HAS-BLED score [OR per point $1.91,95 \%$ CI $1.28-2.84, P<0.01$ ] (Table 4).

Taken together, 97 (17.0\%) of 570 patients receiving apixaban or rivaroxaban had an off-label daily dose at discharge after stroke, with 75 (13.2\%) patients being under-dosed. Under-dosing of rivaroxaban or apixaban was associated with age $\geq 80$ years [OR 2.27, 95\% CI 1.30-3.96, $P<0.01$ ], body weight $\leq 60 \mathrm{~kg}$ [OR $0.21,95 \%$ CI $0.07-0.62, P<0.01$ ], a first episode of AF in-hospital [OR 0.49, 95\% CI 0.25-0.95, $P=0.03$ ], and the HAS-BLED score [OR per point $1.54,95 \%$ CI 1.09-2.16, $P=0.01$ ] (Table 4 online supplement).

Edoxaban was dosed on-label in all four cases prescribed after stroke. An inappropriate daily dose was prescribed in $21(15.7 \%)$ of 134 patients on dabigatran, with $2(1.5 \%)$ patients being over-dosed (receiving $150 \mathrm{mg}$ TD instead of $110 \mathrm{mg}$ TD), with 19 (14.2\%) patients being under-dosed (receiving $110 \mathrm{mg}$ TD instead of $150 \mathrm{mg}$ TD). Prescription of dabigatran $110 \mathrm{mg}$ TD instead of $150 \mathrm{mg}$ TD at hospital discharge was independently associated with congestive heart failure [OR 5.15, 95\% CI 1.49-18.5, $P<0.01$ ] (Table 6 online supplement). Dosing of dabigatran at hospital discharge was in line with present recommendations in 108 $(80.6 \%)$ out of 134 patients [24].

\section{NOAC off-label use}

One of the dabigatran treated patients had an eGFR $<30 \mathrm{ml} /$ $\mathrm{min} / 1.73 \mathrm{~m}^{2}$ at stroke onset as well as at hospital discharge, indicating an off-label use. As none of the apixaban-, rivaroxaban- or edoxaban-treated patients had an eGFR $<15 \mathrm{ml} /$ $\mathrm{min} / 1.73 \mathrm{~m}^{2}$ on admission or at hospital discharge, there was no factor Xa inhibitor off-label use.

\section{Medication changes and outcome in patients on under-dosed rivaroxaban or apixaban at hospital discharge}

One year after the index stroke/TIA, follow-up data on medication were available in 590 (83.3\%) of 708 patients prescribed a NOAC at hospital discharge, including 54 of 75 patients discharged on under-dosed rivaroxaban or apixaban. Assuming an unchanged kidney function at hospital discharge and at 12 months, 37 (68.5\%) of 54 patients prescribed under-dosed rivaroxaban or apixaban at hospital discharge were still on under-dosed rivaroxaban or apixaban, $13(24.1 \%)$ patients were switched to the on-label dose of rivaroxaban or apixaban, $3(5.6 \%)$ were switched to VKA or heparin in therapeutic dose, and one (1.9\%) patient was switched to antiplatelet therapy at 12 months after index stroke. Further information can be found in the Online Supplement.

At 12 months after the index stroke/TIA, recurrent ischemic stroke or TIA, hemorrhagic stroke, myocardial infarction, major bleeding, systemic emboli or all-cause death occurred in $8(14.8 \%)$ of 54 patients on under-dosed apixaban or rivaroxaban at hospital discharge, and in 54 (10.5\%) of 515 patients with on-label prescription of a NOAC at hospital discharge and available follow-up information $(P=0.36)$.

\section{Discussion}

As AF patients with acute ischemic stroke were excluded from phase III trials comparing NOAC to warfarin [25], and registries such as ORBIT AF II [14, 19], GARFIELD-AF 
[26], GLORIA-AF [27], SAKURA AF [28], RE-LY AF [29] and AFNET-2 Register (EORP) [30] did not focus on acute stroke patients with AF, and registries such as PRODAST (NCT02507856) or RASUNOA-prime [31] did not publish such results so far; this post hoc analysis of the prospective multicenter Berlin Atrial Fibrillation Registry is the first detailed report on NOAC off-label use in AF patients with acute ischemic stroke. We demonstrate that $26 \%$ of all registry patients with known AF prescribed a NOAC had an off-label daily dose at stroke onset, with under-dosing in the vast majority of patients (22\%). Of note, apixaban was under-dosed at stroke onset in one out of three patients (33\%), and rivaroxaban was under-dosed in about one out of five patients (22\%). This finding is in line with a German single-center observation based on 254 acute stroke patients with known AF, reporting an off-label dose in one out of three patients on factor Xa inhibitors. [32]

In the Berlin Atrial Fibrillation Registry, under-dosing of apixaban and rivaroxaban at stroke onset was associated with old age, which is in line with prospective AF registries like ORBIT AF II, including only a subset of patients with prior (but not acute) ischemic stroke [14-17]. Compared to on-label dosing, under-dosing of apixaban or rivaroxaban was not significantly associated with stroke severity on hospital admission or at hospital discharge. Our results are not in line with previous reports, stating a lower stroke severity in unselected patients on appropriate NOAC dosing [33]. As an informed consent was requested in the Berlin Atrial Fibrillation Registry, the subsequent selection bias towards minor stroke and TIA patients has to be taken into account.

Under-dosing of rivaroxaban at hospital discharge after acute ischemic stroke or TIA was associated with old age (Table 2), while under-dosing of apixaban was associated with higher $\mathrm{CHA}_{2} \mathrm{DS}_{2}$-VASc as well as HAS-BLED score and inversely associated with lower body weight (Table 3 ). Furthermore, rivaroxaban and apixaban under-dosing at hospital discharge was associated with old age, higher HASBLED score and inversely associated with newly diagnosed AF in-hospital and body weight $\leq 60 \mathrm{~kg}$ (Table 3 online supplement). Of note, one of four patients on under-dosed daily dose of apixaban at discharge did not fulfill one of the three pre-defined dose-reduction criteria, of which two are necessary for prescribing the reduced apixaban dose according to label (Fig. 2 online supplement).

In-hospital treatment of patients with known $\mathrm{AF}$ and an off-label NOAC dosing at stroke onset almost halved the proportion of patients prescribed an off-label NOAC dose ( $26 \%$ on admission vs. $14 \%$ at discharge). However, NOAC off-label dosing was present in $14 \%$ of all registry patients (with either known AF before stroke or a first AF episode inhospital) discharged on a NOAC (Fig. 1). As under-dosing was present in the vast majority of such patients, the fear of secondary hemorrhagic transformation and subsequent clinical worsening might have played an important role. Offlabel NOAC dosing was less likely in stroke/TIA patients with a first episode of AF in-hospital and initiation of NOAC therapy than in patients with known AF before stroke (2.5\% vs $11.2 \%, P<0.01)$.

At 12 months after acute ischemic stroke or TIA, twothirds of all the patients discharged on under-dosed rivaroxaban or apixaban with available follow-up information were still taking the reduced dose of apixaban or rivaroxaban. If compared to patients with on-label NOAC dosing, patients discharged on under-dosed rivaroxaban or apixaban had a similar rate of clinical endpoints (recurrent ischemic stroke or TIA, hemorrhagic stroke, myocardial infarction, major bleeding, systemic embolism or all-cause death) at 12 months after the index stroke or TIA.

Whether continued off-label dosing relates to a higher rate of recurrent stroke during the 3-year follow-up of the Berlin Atrial Fibrillation Registry remains to be analyzed [33]. Nevertheless, off-label dosing of NOACs in stroke patients should be avoided in any case, and awareness of treating physicians is needed. Whether patient identity cards including individual NOAC dose-reduction criteria or detailed documentation in obligatory quality assurance measures may be helpful to reduce off-label dosing of NOACs, has to be investigated.

As no off-label use of factor Xa inhibitors has to be reported, one patient with severe impaired kidney function was prescribed dabigatran at the time of stroke. Keeping in mind that both dabigatran dosing strategies tested in the RE-LY trial are in label in Germany, dabigatran dosing followed recent dosing recommendations in the vast majority of patients [1, 24].

The following limitations have to be addressed: first, providing informed consent by the patient implies a selection bias towards patients with less severe stroke. Second, we cannot exclude that undocumented factors have influenced the physicians' choice of medical stroke prevention in an individual patient. Third, $0.7 \%$ of all registry patients were excluded from the present analysis due to insufficient data. Fourth, as only a few patients were on edoxaban at stroke onset, we cannot draw respective conclusions. Fifth, covering the Berlin area in total, the generalizability of the results is limited. Six, this is an exploratory analysis while the registry was not designed to analyze factors associated with NOAC off-label dosing and the impact on clinical outcome in-hospital.

\section{Conclusions}

Off-label dosing of NOAC therapy in patients with AF is common at onset of acute stroke/TIA, and the vast majority of such patients is under-dosed. Under-dosing of rivaroxaban 


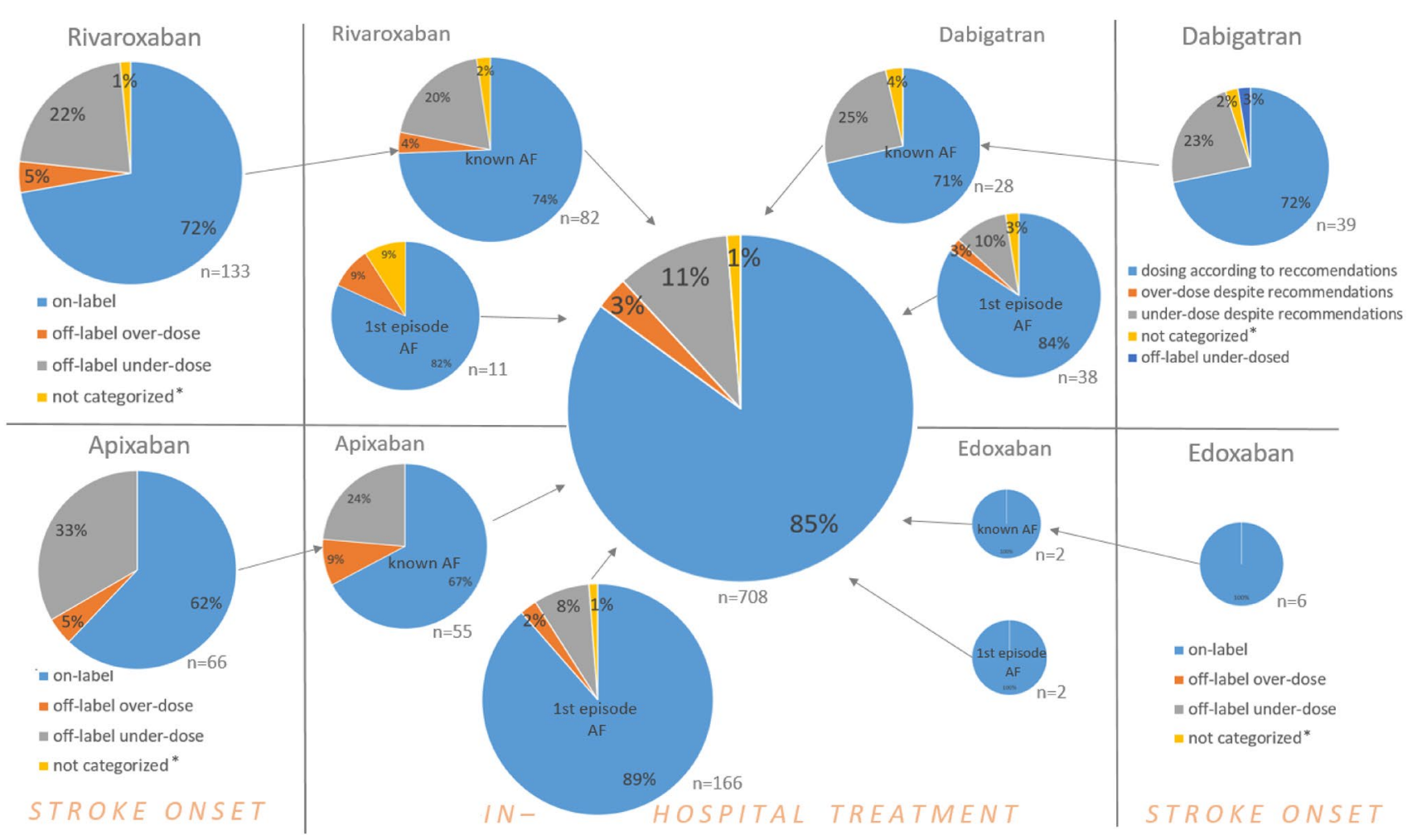

*Not categorized due to missing value (e.g. eGFR or body weight)

Fig. 1 Dosing of NOACs at stroke onset and at hospital discharge in patients with known AF before stroke and in patients with a first episode of AF after acute ischemic stroke/TIA as well as in all AF

or apixaban was more often found in elderly AF patients. In-hospital treatment reduced the number of patients with off-label dosing by half, but one out of ten patients remained off-label under-dosed after in-hospital treatment. Compared to patients with known AF before stroke, off-label dosing was less likely in stroke patients with a first episode of AF in-hospital. Again, under-dosing of rivaroxaban or apixaban at hospital discharge was more often found in elderly AF patients and those with higher HAS-BLED scores. Further efforts are needed to reduce off-label dosing of NOACs in AF patients at risk of stroke.

Supplementary Information The online version contains supplementary material available at https://doi.org/10.1007/s00415-021-10866-2.

Acknowledgements We thank all the members of the study sites and all the members of the study coordinating office at the Center for Stroke Research Berlin, Berlin, Germany.

Funding Open Access funding enabled and organized by Projekt DEAL. This work was supported by an unrestricted research grant from Bayer Vital GmbH, Germany. The authors are solely responsible for the design and conduct of this registry, all analyses, the drafting and editing of the paper and its final contents. patients at hospital discharge. *Not categorized due to missing value (e.g., eGFR or body weight)

Availability of data and material The data that support the findings of this study cannot be shared publicly due to restricted informed consent given by study patients. Reasonable requests may be directed to the corresponding author of the study.

Code availability Not applicable.

\section{Declarations}

Conflicts of interest ST, MO, CK, CF, DZ, CM, BD, JMR, TEH and HCK report no conflict of interests outside the submitted work. DGN has received speaker's honoraria and consulting fees from Bayer, Boehringer Ingelheim, Bristol-Myers-Squibb, Daiichi Sankyo, Novartis and Pfizer. CHN received research grants from German Ministry of Research and Education, German Center for Neurodegenerative Diseases, German Center for cardiovascular Research, and speaker and/or consultation fees from Boehringer Ingelheim, BristolMyers Squibb, Pfizer Pharma, Abbott and W.L. Gore and Associates. JD received a research grant from the Karl and Veronica Carstens Foundation. EV reports that his unmarried partner works for Pfizer. WD reports speaker fees and advisory honoraria from Aimediq, Bayer, Boehringer Ingelheim, Lilly, Medtronic, Pfizer, Sanofi-Aventis, Sphingotec, Vifor Pharma and research support from Vifor Pharma, ZS Pharma outside of the submitted work. AK received speakers honoraria from Bayer, Pfizer and Novartis. PUH reports grants from CharitéUniversitätsmedizin Berlin during study conduct (within MonDAFIS for biometry; member scientific board); research grants from German 
Ministry of Research and Education, German Research Foundation, European Union, Charité - Universitätsmedizin Berlin, Berlin Chamber of Physicians, German Parkinson Society, University Hospital Würzburg, Robert Koch Institute, German Heart Foundation, Federal Joint Committee (G-BA) within the Innovationfond, University Hospital Heidelberg (within RASUNOA-prime; supported by an unrestricted research grant to the University Hospital Heidelberg from Bayer, BMS, Boehringer-Ingelheim, Daiichi Sankyo), University Göttingen (within FIND-AF randomized; supported by an unrestricted research grant to the University Göttingen from Boehringer-Ingelheim), outside the submitted work. ME reports grants from Bayer and fees paid to the Charité from AstraZeneca, Bayer, Boehringer Ingelheim, BristolMyers-Squibb, Ever, Glaxo Smith Kline, MSD, Novartis and Pfizer. $\mathrm{KGH}$ reports speaker's honoraria, consulting fees, lecture honoraria and/or study grants from AstraZeneca, Abbott, Bayer Healthcare, Sanofi, Boehringer Ingelheim, Daiichi Sankyo, Pfizer, Premier Research, Bristol-Myers Squibb, Biotronik, Medronic, Portola, W.L. Gore and Associates and Edwards Lifesciences.

Ethics approval The Charité-Universitätsmedizin Berlin is the sponsor of the investigator-initiated prospective multicenter Berlin Atrial Fibrillation Registry (NCT02306824) which received primary approval from the local Ethics Committee of the Charite-Universitätsmedizin Berlin, Germany in 2014 (EA2/033/14).

Consent to participate Only patients who were able to give informed consent within the in-hospital stay were included.

Consent for publication All included patients gave their consent for publication of the collected data.

Ethical standard statement This chapter does not contain any studies with human participants or animals performed by any of the authors.

Open Access This article is licensed under a Creative Commons Attribution 4.0 International License, which permits use, sharing, adaptation, distribution and reproduction in any medium or format, as long as you give appropriate credit to the original author(s) and the source, provide a link to the Creative Commons licence, and indicate if changes were made. The images or other third party material in this article are included in the article's Creative Commons licence, unless indicated otherwise in a credit line to the material. If material is not included in the article's Creative Commons licence and your intended use is not permitted by statutory regulation or exceeds the permitted use, you will need to obtain permission directly from the copyright holder. To view a copy of this licence, visit http://creativecommons.org/licenses/by/4.0/.

\section{References}

1. Connolly SJ, Ezekowitz MD, Yusuf S, Eikelboom J, Oldgren J, Parekh A, Pogue J, Reilly PA, Themeles E, Varrone J et al (2009) Dabigatran versus warfarin in patients with atrial fibrillation. $\mathrm{N}$ Engl J Med 361(12):1139-1151

2. Patel MR, Mahaffey KW, Garg J, Pan G, Singer DE, Hacke W, Breithardt G, Halperin JL, Hankey GJ, Piccini JP et al (2011) Rivaroxaban versus warfarin in nonvalvular atrial fibrillation. $\mathrm{N}$ Engl J Med 365(10):883-891

3. Granger CB, Alexander JH, McMurray JJ, Lopes RD, Hylek EM, Hanna M, Al-Khalidi HR, Ansell J, Atar D, Avezum A et al (2011) Apixaban versus warfarin in patients with atrial fibrillation. N Engl J Med 365(11):981-992
4. Giugliano RP, Ruff CT, Braunwald E, Murphy SA, Wiviott SD, Halperin JL, Waldo AL, Ezekowitz MD, Weitz JI, Špinar J et al (2013) Edoxaban versus warfarin in patients with atrial fibrillation. N Engl J Med 369(22):2093-2104

5. Xian Y, Xu H, O'Brien EC, Shah S, Thomas L, Pencina MJ, Fonarow GC, Olson DM, Schwamm LH, Bhatt DL et al (2019) Clinical effectiveness of direct oral anticoagulants vs warfarin in older patients with atrial fibrillation and ischemic stroke: findings from the patient-centered research into outcomes stroke patients prefer and effectiveness research (PROSPER) study. JAMA Neurol 76(10):1192-1202

6. Hindricks G, Potpara T, Dagres N, Arbelo E, Bax JJ, BlomströmLundqvist C, Boriani G, Castella M, Dan GA, Dilaveris PE et al (2020) ESC Guidelines for the diagnosis and management of atrial fibrillation developed in collaboration with the European Association of Cardio-Thoracic Surgery (EACTS). Eur Heart J

7. January CT, Wann LS, Calkins H, Chen LY, Cigarroa JE, Cleveland JC Jr, Ellinor PT, Ezekowitz MD, Field ME, Furie KL et al (2019) 2019 AHA/ACC/HRS focused update of the 2014 AHA/ ACC/HRS guideline for the management of patients with atrial fibrillation: a report of the American College of Cardiology/ American Heart Association Task Force on clinical practice guidelines and the heart rhythm society in collaboration with the society of thoracic surgeons. Circulation 140(2):e125-e151

8. (AWMF) AdWMF: S3-Leitlinie Sekundärprophylaxe ischämischer Schlaganfall und transitorische ischämische Attacke

9. Haeusler KG, Tütüncü S, Kunze C, Schurig J, Malsch C, Harder J, Wiedmann S, Dimitrijeski B, Ebinger M, Hagemann G et al (2019) Oral anticoagulation in patients with atrial fibrillation and acute ischaemic stroke: design and baseline data of the prospective multicentre Berlin Atrial Fibrillation Registry. Europace 21(11):1621-1632

10. Tanislav C, Allendörfer J, Pfeilschifter W, Fuest S, Stein M, Meyer M, Reuter I, Kaps M, Misselwitz B (2018) One decade of oral anticoagulation in stroke patients: results from a large country-wide hospital-based registry. Int J Stroke 13(3):308-312

11. Hohnloser SH, Basic E, Nabauer M (2019) Uptake in antithrombotic treatment and its association with stroke incidence in atrial fibrillation: insights from a large German claims database. Clin Res Cardiol 108(9):1042-1052

12. Steinberg BA, Gao H, Shrader P, Pieper K, Thomas L, Camm AJ, Ezekowitz MD, Fonarow GC, Gersh BJ, Goldhaber S et al (2017) International trends in clinical characteristics and oral anticoagulation treatment for patients with atrial fibrillation: results from the GARFIELD-AF, ORBIT-AF I, and ORBIT-AF II registries. Am Heart J 194:132-140

13. Steffel J, Verhamme P, Potpara TS, Albaladejo P, Antz M, Desteghe L, Haeusler KG, Oldgren J, Reinecke H, RoldanSchilling V et al (2018) The 2018 European Heart Rhythm Association Practical Guide on the use of non-vitamin K antagonist oral anticoagulants in patients with atrial fibrillation. Eur Heart J 39(16):1330-1393

14. Steinberg BA, Shrader P, Thomas L, Ansell J, Fonarow GC, Gersh BJ, Kowey PR, Mahaffey KW, Naccarelli G, Reiffel J et al (2016) Off-label dosing of non-vitamin $\mathrm{k}$ antagonist oral anticoagulants and adverse outcomes: the ORBIT-AF II Registry. J Am Coll Cardiol 68(24):2597-2604

15. Steinberg BA, Washam JB (2017) Appropriate dosing of nonvitamin $\mathrm{K}$ antagonist oral anticoagulants for stroke prevention in atrial fibrillation. Trends Cardiovasc Med 27(8):567-572

16. Miyazaki M, Matsuo K, Uchiyama M, Nakamura Y, Sakamoto Y, Misaki M, Tokura K, Jimi S, Okamura K, Adachi S et al (2020) Inappropriate direct oral anticoagulant dosing in atrial fibrillation patients is associated with prescriptions for outpatients rather than inpatients: a single-center retrospective cohort study. J Pharm Health Care Sci 6:2 
17. Ablefoni K, Buchholz A, Ueberham L, Hilbert S, Dagres N, Husser D, Hindricks G, Bollmann A (2019) Initial rivaroxaban dosing in patients with atrial fibrillation. Clin Cardiol 42(10):873-880

18. Wang KL, Lopes RD, Patel MR, Büller HR, Tan DS, Chiang CE, Giugliano RP (2019) Efficacy and safety of reduced-dose non-vitamin $\mathrm{K}$ antagonist oral anticoagulants in patients with atrial fibrillation: a meta-analysis of randomized controlled trials. Eur Heart J 40(19):1492-1500

19. Steinberg BA, Shrader P, Pieper K, Thomas L, Allen LA, Ansell J, Chan PS, Ezekowitz MD, Fonarow GC, Freeman JV et al (2018) Frequency and outcomes of reduced dose non-vitamin $\mathrm{K}$ antagonist anticoagulants: results from ORBIT-AF II (The Outcomes Registry for Better Informed Treatment of Atrial Fibrillation II). J Am Heart Assoc 7(4)

20. Amarenco P, Haas S, Hess S, Kirchhof P, Lambelet M, Bach M, Turpie AGG, Camm AJ (2019) Outcomes associated with nonrecommended dosing of rivaroxaban: results from the XANTUS study. Eur Heart J Cardiovasc Pharmacother 5(2):70-79

21. Hatano S (1976) Experience from a multicentre stroke register: a preliminary report. Bull World Health Organ 54(5):541-553

22. Brott T, Adams HP Jr, Olinger CP, Marler JR, Barsan WG, Biller J, Spilker J, Holleran R, Eberle R, Hertzberg V et al (1989) Measurements of acute cerebral infarction: a clinical examination scale. Stroke 20(7):864-870

23. van Swieten JC, Koudstaal PJ, Visser MC, Schouten HJ, van Gijn J (1988) Interobserver agreement for the assessment of handicap in stroke patients. Stroke 19(5):604-607

24. Ingelheim B (2019) Fachinformation Pradaxa $150 \mathrm{mg}$ Hartkapseln

25. Ruff CT, Giugliano RP, Braunwald E, Hoffman EB, Deenadayalu N, Ezekowitz MD, Camm AJ, Weitz JI, Lewis BS, Parkhomenko A et al (2014) Comparison of the efficacy and safety of new oral anticoagulants with warfarin in patients with atrial fibrillation: a meta-analysis of randomised trials. Lancet 383(9921):955-962
26. Haas S (2015) im Namen des G-AFSC: [GARFIELD-AF - First data on healthcare of patients with atrial fibrillation in Germany]. Dtsch Med Wochenschr 140(Suppl 1):S13-14

27. Huisman MV, Rothman KJ, Paquette M, Teutsch C, Diener HC, Dubner SJ, Halperin JL, Ma C, Zint K, Elsaesser A et al (2015) Antithrombotic treatment patterns in patients with newly diagnosed nonvalvular atrial fibrillation: the GLORIA-AF Registry Phase II. Am J Med 128(12):1306-1313.e1301

28. Okumura Y, Yokoyama K, Matsumoto N, Tachibana E, Kuronuma K, Oiwa K, Matsumoto M, Kojima T, Hanada S, Nomoto $\mathrm{K}$ et al (2017) Current use of direct oral anticoagulants for atrial fibrillation in Japan: findings from the SAKURA AF Registry. J Arrhythm 33(4):289-296

29. Paikin JS, Haroun MJ, Eikelboom JW (2011) Dabigatran for stroke prevention in atrial fibrillation: the RE-LY trial. Expert Rev Cardiovasc Ther 9(3):279-286

30. Boriani G, Proietti M, Laroche C, Fauchier L, Marin F, Nabauer M, Potpara T, Dan GA, Kalarus Z, Tavazzi L et al (2019) Association between antithrombotic treatment and outcomes at 1-year follow-up in patients with atrial fibrillation: the EORP-AF General Long-Term Registry. Europace 21(7):1013-1022

31. Haas K, Purrucker JC, Rizos T, Heuschmann PU, Veltkamp R (2019) Rationale and design of the Registry of Acute Stroke Under Novel Oral Anticoagulants-prime (RASUNOA-prime). Eur Stroke J 4(2):181-188

32. Stoll S, Macha K, Marsch A, Gerner ST, Siedler G, Fröhlich K, Volbers B, Strasser EF, Schwab S, Kallmünzer B (2020) Ischemic stroke and dose adjustment of oral factor Xa inhibitors in patients with atrial fibrillation. J Neurol 267(7):2007-2012

33. Hellwig S, Grittner U, Audebert H, Endres M, Haeusler KG (2018) Non-vitamin K-dependent oral anticoagulants have a positive impact on ischaemic stroke severity in patients with atrial fibrillation. EP Europace 20(4):569-574

\title{
Authors and Affiliations
}

\author{
Serdar Tütüncü ${ }^{1} \cdot$ Manuel Olma $^{1,2} \cdot$ Claudia Kunze $^{1}$. Joanna Dietzel ${ }^{1}$. Johannes Schurig ${ }^{1}$. \\ Cornelia Fiessler $^{3}$. Carolin Malsch ${ }^{3,4} \cdot$ Tobias Eberhard Haas $^{3}$ - Boris Dimitrijeski ${ }^{5}$. Wolfram Doehner ${ }^{1,6}$. \\ Georg Hagemann $^{7} \cdot$ Frank Hamilton $^{8} \cdot$ Martin Honermann $^{9} \cdot$ Gerhard Jan Jungehulsing $^{10}$. Andreas Kauert ${ }^{11}$. \\ Hans-Christian Koennecke ${ }^{12}$. Bruno-Marcel Mackert ${ }^{8} \cdot$ Darius Nabavi $^{5}$. Christian H. Nolte ${ }^{1,2,19,20}$. \\ Joschua Mirko Reis ${ }^{3} \cdot$ Ingo Schmehl $^{13} \cdot$ Paul Sparenberg $^{13} \cdot$ Robert Stingele $^{14} \cdot$ Enrico Völzke $^{15}$. \\ Carolin Waldschmidt ${ }^{16}$. Daniel Zeise-Wehry ${ }^{17}$. Peter U. Heuschmann ${ }^{3,4}$. Matthias Endress $s^{1,2,18,19,20}$. \\ Karl Georg Haeusler ${ }^{21}$
}

1 Center for Stroke Research Berlin, CharitéUniversitätsmedizin Berlin, Berlin, Germany

2 Department of Neurology, Charité-Universitätsmedizin Berlin, Berlin, Germany

3 Institute of Clinical Epidemiology and Biometry, University Würzburg, Würzburg, Germany

4 Comprehensive Heart Failure Center, Clinical Trial Centre Würzburg, University of Würzburg, University Hospital Würzburg, Würzburg, Germany

5 Department of Neurology, Vivantes Klinikum Neukölln, Berlin, Germany

6 BCRT-Berlin Institute of Health Center for Regenerative Therapies, and Department of Cardiology (Virchow Klinikum), Charité-Universitätsmedizin Berlin, German
Centre for Cardiovascular Research (DZHK), Partner Site Berlin, Berlin, Germany

7 Department of Neurology, Helios Klinik Berlin-Buch, Berlin, Germany

8 Department of Neurology, Vivantes Auguste-Viktoria-Klinikum, Berlin, Germany

9 Department of Neurology, Vivantes Klinikum Spandau, Berlin, Germany

10 Department of Neurology, Jüdisches Krankenhaus Berlin, Berlin, Germany

11 Department of Neurology, Evangelisches Krankenhaus Königin Elisabeth Herzberge, Berlin, Germany

12 Department of Neurology, Vivantes Klinikum im Friedrichshain, Berlin, Germany 
13 Department of Neurology, BG Klinikum Unfallkrankenhaus Berlin, Berlin, Germany

14 Department of Neurology, German Red Cross Hospital Berlin Köpenick, Berlin, Germany

15 Department of Neurology, Schlosspark-Klinik Berlin, Berlin, Germany

16 Department of Neurology, Vivantes Humboldt-Klinikum, Berlin, Germany

17 Department of Neurology, Park-Klinik Weissensee, Berlin, Germany
18 German Center for Neurodegenerative Diseases (DZNE), Partner site Berlin, Berlin, Germany

19 German Center for Cardiovascular Diseases (DZHK), Partner site Berlin, Berlin, Germany

20 Berlin Institute of Health (BIH), Berlin, Germany

21 Department of Neurology, Universitätsklinikum Würzburg, Würzburg, Germany 